\title{
On Target Identification Based on Group Decision Method
}

\author{
Wanhai Guo \& Zhenxing Liu \& Songtao Liu \& Longtai Wang \\ Dalian Naval Academy, Dalian 116018, China
}

Keywords: identification; grey system; operation method.

\begin{abstract}
In order to solve the problem of the group decision-making of target identification, using the method of the group decision-making Based on the grey multi-attribute risk. An example illustrates the validity and efficiency of the grey multi-attribute risk group decision-making. Breaks through the limitation in previous measuring method, put forward the operation method is convenient, the more close to the troops and training practice.
\end{abstract}

\section{Introduction}

Research on the target threat identification was carried on since 70's in last century in the world. Continuously go deep into along with the information warfare theories, process several years of research and fulfillment, combine perfect various research means and method which gradually advanced have already formed more perfect theories and method.

But along with the navy equipment system construction of development and performance task category diversification, the constitute of target become also more huge quantitatively, the platform type get more and more and the command relationship of target threat identification also go into more complicatedly.

\section{The command relationship of target threat identification}

The command relationship of target threat identification can is divided into following few kinds:

(1) Centralized command and Distributed execution. The intelligence station get contact only with decision layer, the decision layer deploys task and battle information to each aspect commander, and the aspect commander command a combat by oneself.

(2) Centralized command and control. The intelligence station gets contact with decision layer and every commander, commander hierarchical command and feedback in time.

(3) Coordinated command and control. Decision layer cooperate with each aspect commander and be in conjunction with action, to command branch and weapon system centralized.

(4) Distributed command and Separate synchronization execution. The decision layer deploys task, each branch combats by oneself battle.

The fourth only applies with the operational command of target threat identification under the special circumstances, and take no advisement because its limitation. Here we take the first, the second, the third command relationship of target threat identification to carry on a comparison.

The optimization to command relationship of target threat identification adopts gray risk group decision-making decision methods.

\section{Description of the grey multi-attribute risk group decision-making method}

The brief characteristics of the grey multi-attribute risk group decision-making method is that the attribute value of solution is random variable, it varies with various estate, the decision maker cannot indeed know its estate in the future, but can give various possibility estate. The decision maker give different hobby attribute value to various estate according to the own knowledge, experience and preference. 
Let the solution set of grey multi-attribute risk group decision-making $A=\left\{A_{1}, A_{2}, \ldots, A_{n}\right\}$, The decision group set $E=\left\{e_{1}, e_{2}, \ldots, e_{q}\right\}(q \geq 2)$, where $e_{s}$ said first s decision makers, its corresponding weigh $\lambda_{s}$, meet $0 \leq \lambda_{s} \leq 1, \sum_{s=1}^{q} \lambda_{s}=1$. The decision attribute set $\mu=\left\{\mu_{1}, \mu_{2}, \ldots \mu_{m}\right\}$, attribute $\mu_{j}$ weight $\omega_{j}$, meet $0 \leq \omega_{j} \leq 1, \sum_{j=1}^{m} \omega_{j}=1$,weight vector $w=\left(\omega_{1}, \omega_{2}, \ldots, \omega_{m}\right)$ unknown, for each decision attribute $\mu_{j}$ has $l$ possible states $0 \leq p_{t j}^{s} \leq 1(1 \leq t \leq l)$, for each decision maker $e_{s}$ in the attribute $\mu_{j}$ state $\theta_{t}$ occurrence Probability $0 \leq p_{t j}^{s} \leq 1(1 \leq t \leq l), \sum_{t=1}^{l} p_{t j}^{s}=1$, attribute values of solution $a_{i j t}^{s}(\otimes) \in\left[\underline{a}_{i j t}^{s}, \bar{a}_{i j t}^{s}\right]$. Decision maker $e_{s}$ give risk decision information of each state as shown in Table 1. In the known decision maker $e_{s}(s=1,2, \ldots, q)$ risk decision information, need for comprehensive assessment and sort.

Table 1. Decision maker $e_{s}$ risk decision table $E_{s}(\otimes)$

\begin{tabular}{|c|c|c|c|c|c|c|c|c|c|c|c|c|c|}
\hline \multirow[b]{2}{*}{$\begin{array}{l}\text { Identification } \\
\text { relationship }\end{array}$} & \multicolumn{4}{|c|}{$\mu_{1}$} & \multicolumn{4}{|c|}{$\mu_{2}$} & $\ldots$ & \multicolumn{4}{|c|}{$\mu_{m}$} \\
\hline & $\begin{array}{l}\theta_{1} \\
p_{11}^{s}\end{array}$ & $\begin{array}{l}\theta_{2} \\
p_{21}^{s}\end{array}$ & $\begin{array}{l}\ldots \\
\cdots\end{array}$ & $\begin{array}{l}\theta_{l} \\
p_{l 1}^{s}\end{array}$ & $\begin{array}{l}\theta_{1} \\
p_{12}^{s}\end{array}$ & $\begin{array}{l}\theta_{2} \\
p_{22}^{s}\end{array}$ & $\begin{array}{l}\ldots \\
\cdots\end{array}$ & $\begin{array}{l}\theta_{l} \\
p_{l 2}^{s}\end{array}$ & $\begin{array}{l}\ldots \\
\cdots\end{array}$ & $\begin{array}{c}\theta_{1} \\
p_{1 m}^{s}\end{array}$ & $\begin{array}{c}\theta_{2} \\
p_{2 m}^{s}\end{array}$ & $\begin{array}{l}\ldots \\
\ldots\end{array}$ & $\begin{array}{l}\theta_{l} \\
p_{l m}^{s}\end{array}$ \\
\hline$A_{1}$ & $a_{111}^{s}(\otimes)$ & $a_{112}^{s}(\otimes)$ & $\ldots$ & $a_{11 l}^{s}(\otimes)$ & $a_{121}^{s}(\otimes)$ & $a_{122}^{s}(\otimes)$ & $\ldots$ & $a_{12 l}^{s}(\otimes)$ & $\ldots$ & $a_{1 m 1}^{s}(\otimes)$ & $a_{1 m 2}^{s}(\otimes)$ & $\ldots$ & $a_{1 m l}^{s}(\otimes)$ \\
\hline$A_{2}$ & $a_{211}^{s}(\otimes)$ & $a_{212}^{s}(\otimes)$ & $\ldots$ & $a_{21 l}^{s}(\otimes)$ & $a_{221}^{s}(\otimes)$ & $a_{222}^{s}(\otimes)$ & $\ldots$ & $a_{22 l}^{s}(\otimes)$ & $\ldots$ & $a_{2 m 1}^{s}(\otimes)$ & $a_{2 m 2}^{s}(\otimes)$ & $\cdots$ & $a_{2 m l}^{s}(\otimes)$ \\
\hline $\begin{array}{l}\cdots \\
A_{n}\end{array}$ & $a_{n 11}^{s}(\otimes)$ & $a_{n 12}^{s}(\otimes)$ & $\ldots$ & $a_{n 1 l}^{s}(\otimes)$ & $a_{n 21}^{s}(\otimes)$ & $\begin{array}{c}\cdots \\
a_{n 22}^{s}(\otimes)\end{array}$ & $\ldots$ & $a_{n 2 l}^{s}(\otimes)$ & $\begin{array}{l}\ldots \\
\ldots\end{array}$ & $a_{n m 1}^{s}(\otimes)$ & $\begin{array}{c}\cdots \\
a_{n m 2}^{s}(\otimes)\end{array}$ & $\ldots$ & $a_{n m l}^{s}(\otimes)$ \\
\hline
\end{tabular}

Data processing and weight calculation here omit, the decision algorithm:

Step 1. In order to comprehensively considering the preference of decision maker, the decision matrix $y^{s}(\otimes)(s=1,2, \ldots, q)$ to rewrite on decision solution $A_{i}$ standardization decision matrix

$$
\begin{aligned}
& R^{i}(\otimes)(i=1,2, \ldots, n) . \\
& R^{i}(\otimes)=\left(\begin{array}{l}
{\left[\underline{y}_{i 1}^{1}, \bar{y}_{i 1}^{1}\right]\left[\underline{y}_{i 2}^{1}, \bar{y}_{i 2}^{1}\right] \ldots\left[\underline{y}_{i m}^{1}, \bar{y}_{i m}^{1}\right]} \\
{\left[\underline{y}_{i 1}^{2}, \bar{y}_{i 1}^{2}\right]\left[\underline{y}_{i 2}^{2}, \bar{y}_{i 2}^{2}\right] \ldots\left[\underline{y}_{i m}^{2}, \bar{y}_{i m}^{2}\right]} \\
{\left[\underline{y}_{i 1}^{q}, \bar{y}_{i 1}^{q}\right]\left[\underline{y}_{i 2}^{q}, \bar{y}_{i 2}^{q}\right] \ldots\left[\underline{y}_{i m}^{q}, \bar{y}_{i m}^{q}\right]}
\end{array}\right)
\end{aligned}
$$

Step 2. To construct ideal matrix $F(\otimes)=\left(f_{s j}(\otimes)\right)_{q \times m}$ and the negative ideal matrix $G(\otimes)=\left(g_{s j}(\otimes)\right)_{q \times m}$, where

$$
\begin{aligned}
& f_{s j}(\otimes) \in\left[\underline{f}_{s j}, \bar{f}_{s j}\right] ; g_{s j}(\otimes) \in\left[\underline{g}_{s j}, \bar{g}_{s j}\right], \\
& j=1,2, \ldots m ; s=1,2, \ldots q
\end{aligned}
$$

Note

$\underline{f}_{s j}=\max _{1 \leq i \leq n}\left(\underline{y}_{i j}^{s}\right), \bar{f}_{s j}=\max _{1 \leq i \leq n}\left(\bar{y}_{i j}^{s}\right)$

$\underline{g}_{s j}=\min _{1 \leq i \leq n}\left(\underline{y}_{i j}^{s}\right), \bar{g}_{s j}=\min _{1 \leq i \leq n}\left(\bar{y}_{i j}^{s}\right)$

Step 3. Calculation of Euclidean distance, any solution matrix $R^{i}(\otimes)(i=1,2, \ldots, n)$ and ideal matrix $F(\otimes)=\left(f_{s j}(\otimes)\right)_{q \times m}$ and negative ideal matrix $G(\otimes)=\left(g_{s j}(\otimes)\right)_{q \times m}$ distance respectively 


$$
\begin{aligned}
& d_{i}^{+}=d\left(R^{i}(\otimes), F(\otimes)\right)=\left\{\sum_{s=1}^{q} \lambda_{s} \sum_{j=1}^{m} \omega_{j}\left[\left|\underline{y}_{i j}^{s}-\underline{f}_{s j}\right|^{2}+\left|\bar{y}_{i j}^{s}-\bar{f}_{s j}\right|^{2}\right]\right\}^{\frac{1}{2}} \\
& d_{i}^{-}=d\left(R^{i}(\otimes), G(\otimes)\right)=\left\{\sum_{s=1}^{q} \lambda \sum_{j=1}^{m} \omega_{j}\left[\left|\underline{y}_{i j}^{s}-\underline{g}_{s j}\right|^{2}+\left|\bar{y}_{i j}^{s}-\bar{g}_{s j}\right|^{2}\right]\right\}^{\frac{1}{2}}
\end{aligned}
$$

$d_{i}^{+}$More big, said the decision solution $A_{i}$ closer to the ideal solution, solution $A_{i}$ more excellent, $d_{i}^{-}$meaning exactly opposite. Therefore, the optimal decision solution should be as close as possible to the ideal solution, at the same time as far as possible away from the negative ideal solution. The solution $A_{i}$ the relative membership degree to the ideal plan $\mu_{i}$, and the solution $A_{i}$ has the relative membership degree to the negative ideal plan $1-\mu_{i}$.

Establishment of the model

$$
\left(M_{2}\right) \min F\left(\mu_{i}\right)=\mu_{i}^{2} d_{i}^{+2}+\left(1-\mu_{i}\right)^{2} d_{i}^{-2}, i=1,2, \ldots, n
$$

The optimal solution of the model

$$
\mu_{i}=\frac{1}{1+\left(d_{i}^{+2} / d_{i}^{-2}\right)}
$$

The solution sort according to the size of $\mu_{i}(i=1,2, \ldots, n)$, the greater that the optimal solution.

\section{Target identification command process decision analysis}

In this paper, three kinds of radar radiating source identification command flow are analyzed

$A_{1}, A_{2}, A_{3}$ Said different command relationships corresponding to different command flow $1,2,3$;

The three main attributes of each process

$\mu_{1}$ System responses to rapid degree.

$\mu_{2}$ Command and control orders accuracy.

$\mu_{3}$ Redundancy and complexity.

Three decision makers from the different sector $e_{1}, e_{2}, e_{3}$ presented respectively the assessment data for each command flow, and assuming the weight vector $\lambda=(0.3,0.4,0.3)$. Then we determine the optimal command flow according to the above steps. Calculation process is slightly here.

(1) Let the table into the no risk decision matrix through the expected value calculation formula, get standardized no risk matrix sequence through the standardized treatment $Y^{s}(\otimes)(s=1,2,3)$

(2) Three main attributes weight vector $\mathrm{w}=(0.2,0.3,0.5)$ of command flow can be obtained by the relevant model.

(3) The decision matrix $Y^{s}(\otimes)(s=1,2,3)$ can be written as standardization decision matrix $R^{i}(\otimes)(i=1,2,3)$ about solution $A_{i}$.

(4) Construct ideal matrix $F(\otimes)=\left(f_{s j}(\otimes)\right)_{q \times m}$ and negative ideal matrix $G(\otimes)=\left(g_{s j}(\otimes)\right)_{q \times m}$

(5) Calculate the membership degree of each command flow on ideal solution by model $\left(M_{2}\right)$

$$
\mu_{1}=0.432,{ }^{\mu_{2}}=0.518,{ }^{\mu_{3}}=0.352
$$

The sort of the three command flow $A_{2} \succ A_{1} \succ A_{3}$, therefore the optimal command flow solution $A_{2}$, and the optimal solution is the first command relation corresponding to the command flow. 


\section{Conclusion}

In this paper, we carry on an optimization to command relationship of target identification through gray risk group decision-making decision methods, and get its related conclusion. We give a new way to solve the similar problem, in the meantime provide a kind optimization method which of clear concept and operation easily.

\section{References}

[1]. Ganonlay. Multiple Platform Sensor Integration Model:ULSIM Computer Program [J]. ADA 079951, Naval Research Laboratory, 31, Dec. 1999:1-62

[2]. D.LHall. Mathematical Techniques in Multisensor Data Fusoion[M]. Artech House, Boston, Lonton, 1992

[3]. J.Llinas, E.Waltz. Multisensor Data Fusion [M]. Artech House, Norwood, Massachusetts, 1990

[4]. L. James, Fisher\&David P. Casasent. Fast JPDA Multi-target Tracking Alogorithm [J].Applied Optics, Vol. 28, No. 2, 1989:371-376

[5]. Waltz L. Multiple Sensor Data Fusion [J]. Norwood: A-rtech House, 1990. 